\title{
On Ocneanu's theory of asymptotic inclusions \\ for subfactors, topological quantum field theories and quantum doubles
}

\author{
DAvid E. Evans \\ Department of Mathematics \\ University of Wales Swansea \\ Singleton Park, Swansea SA2 8PP, Wales, U.K. \\ YASUYUKI KAWAHIGASHI \\ Department of Mathematical Sciences \\ University of Tokyo, Hongo, Tokyo, 113, JAPAN
}

\begin{abstract}
A fully detailed account of Ocneanu's theorem is given that the Hilbert space associated to the two-dimensional torus in a Turaev-Viro type $(2+1)$ dimensional topological quantum field theory arising from a finite depth subfactor $N \subset M$ has a natural basis labeled by certain $M_{\infty}-M_{\infty}$ bimodules of the asymptotic inclusion $M \vee\left(M^{\prime} \cap M_{\infty}\right) \subset M_{\infty}$, and moreover that all these bimodules are given by the basic construction from $M \vee\left(M^{\prime} \cap M_{\infty}\right) \subset M_{\infty}$ if the fusion graph is connected. This Hilbert space is an analogue of the space of conformal blocks in conformal field theory. It is also shown that after passing to the asymptotic inclusions we have $S$ - and $T$-matrices, analogues of the Verlinde identity and Vafa's result on roots of unity. It is explained that the asymptotic inclusions can be regarded as a subfactor analogue of the quantum double construction of Drinfel'd. These claims were announced by A. Ocneanu in several talks, but he has not published his proofs, so details are given here along the lines outlined in his talks.
\end{abstract}

\section{Introduction}

The subfactor theory initiated by V. F. R. Jones in [14] and his celebrated knot invariant [15] have attracted much attention, both in the operator algebra theory 
itself and in its relations to quantum group theory, conformal field theory, and 3dimensional topological quantum field theory. A. Ocneanu has announced a striking theory relating these various fields in several recent talks [30], [31], [32], [34], [35], but unfortunately he has not published proofs of his important results. Our aim in this paper is to give full proofs of his recent claims based on his lectures [31], [34] and our earlier efforts [11], [16], [17], [20] about his unpublished theory. Proofs of irreducibility of three kinds of bimodules (Theorems 2.1, 4.1, and 4.2) in this paper are by us. Most of the pictures are taken from [31], [34].

Ocneanu [26] introduced a new algebraic/combinatorial notion a paragroup as an invariant for subfactors of the approximately finite dimensional (AFD) $\mathrm{II}_{1}$ factor with finite Jones index and finite depth. (Also see [27], [28], [16], [17], [19], [20] for more information on paragroups.) He later claimed in [29] that his axioms for paragroups are essentially equivalent to those for combinatorial initial data, the quantum $6 j$ symbols generalizing the Kirillov-Reshetikhin quantum $6 j$-symbols for $\mathcal{U}_{q}\left(s l_{2}\right)$ [22], in the Turaev-Viro type topological quantum field theory (TQFT) in dimension $2+1$ [42] in the sense of Atiyah [1]. He has not published his proof of this equivalence, but a complete proof was given by us in [11] along the lines outlined in his several talks. His more recent idea is that the following three constructions, in algebra, topology, and analysis respectively, give different machinery realizing the same concept.

1. The quantum double construction of Drinfel'd [8]

2. Turaev-Viro type 3-dimensional TQFT based on triangulations [42]

3. Ocneanu's asymptotic inclusion of a subfactor [26], [28]

Furthermore, the third construction also corresponds to the central sequence subfactor [26], [28], which is more analytic. (These constructions of subfactors were introduced by Ocneanu for his analytic classification of subfactors in [26] first. See [18], [21] for more on these constructions in the operator algebra theory. The analytic classification theory of subfactors was completed by S. Popa in [37], [38], [39].) The relations among the three constructions will be clarified in detail in this paper.

In the topological quantum field theory, the Hilbert space $H_{S^{1} \times S^{1}}$ associated to the 2 -torus $S^{1} \times S^{1}$ has a very important meaning, and it is an analogue of the space of conformal blocks in conformal field theory (CFT). Ocneanu sketched a proof of a statement in [34] that the Hilbert space $H_{S^{1} \times S^{1}}$ in the TQFT arising from a subfactor $N \subset M$ with finite index and finite depth has a natural basis labeled by certain $M_{\infty^{-}}$ $M_{\infty}$ bimodules given by the asymptotic inclusion $M \vee\left(M^{\prime} \cap M_{\infty}\right) \subset M_{\infty}$, and that all these bimodules are given by the basic constructions from $M \vee\left(M^{\prime} \cap M_{\infty}\right) \subset M_{\infty}$ if the so-called fusion graph is connected. A proof of this claim is given here.

We also discuss relations of the asymptotic inclusions to rational conformal field theory (RCFT) in the sense of Moore-Seiberg [25]. In the paper [3], de Boer-Goeree tried to generate combinatorial data satisfying the Moore-Seiberg axioms from general paragroups without much success. Ocneanu claimed that one can construct such data after passing to the asymptotic inclusion from the initial subfactor, in which case we 
have $S$ - and $T$-matrices, and analogues of the results of C. Vafa [43] and E. Verlinde [44] hold, as explained below.

A paragroup is regarded as a certain kind of quantization of an ordinary finite group. If a paragroup is really a finite group, it is shown that the asymptotic inclusion gives the dual of the quantum double of the function algebra on the group in the sense of Drinfel'd [8]. Because of this fact, the asymptotic inclusion can be regarded as an analogue of the quantum double construction for paragroups.

This work was completed while the second author visited the University College of Swansea in September/October of 1993 with the financial support of SERC and the Higher Education Funding Council for Wales-Research. He thanks University College of Swansea for its hospitality. We thank D. Bisch for helpful communications, A. Ocneanu for giving us a copy of notes of [31] and comments on this manuscript including a communication of an error in our original manuscript, and $\mathrm{F}$. $\mathrm{Xu}$ for showing us [33] and the notes of [34].

\section{Hilbert spaces for labeled surfaces and surface bimodules}

We start with an AFD type $\mathrm{II}_{1}$ subfactor $N \subset M$ with finite index and finite depth. As usual [11], [26] [28], [29], [31], we deal with the finite system $\mathcal{M}$ of (four kinds of) bimodules arising from the "fundamental bimodule" ${ }_{N} M_{M}$ and tensor product operations. That is, we have a (non-commutative) fusion algebra of bimodules with the tensor product operation and the quantized $6 j$-symbols. (See [36] for the basic theory of bimodules over $\mathrm{II}_{1}$ factors.)

We work on several types of labeling of topological objects in Ocneanu's theory. Assign certain labels given by operator algebras, bimodules, and intertwiners to graphical objects such as vertices, edges, and triangles respectively. Consider oriented triangulated surfaces (possibly with boundaries). Assign a label of an algebra $N$ or $M$ to each vertex. Next assign a label of a bimodule to an oriented edge so that the left- and right-acting algebras of the bimodule coincide with the ones assigned

to vertices. A labeled edge is identified with the edge with reversed orientation and labeling by the conjugate bimodule, as in Figure 2.1.

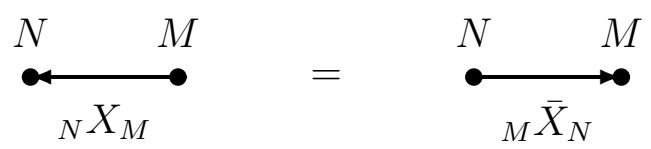

Figure 2.1

Next assign an intertwiner to a triangle with labeled edges so that the intertwiner 
maps from a tensor product of two bimodule to the other as in Figure 2.2, where $\xi \in \operatorname{Hom}\left({ }_{N} X \otimes_{M} Y_{M},{ }_{N} Z_{M}\right)$.

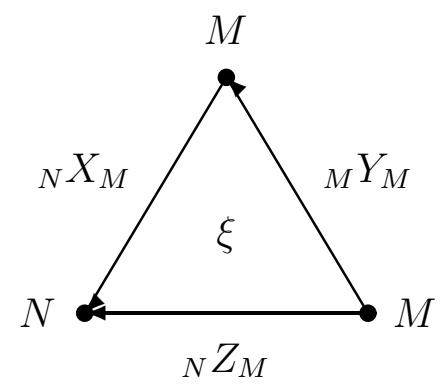

Figure 2.2

After rotating Figure 2.2 by 120 degrees in the counterclockwise orientation, we get the right diagram in Figure 2.3. Identify the labeled diagram in Figure 2.2 with the right labeled diagram in Figure 2.3 using Frobenius reciprocity [29], [47].
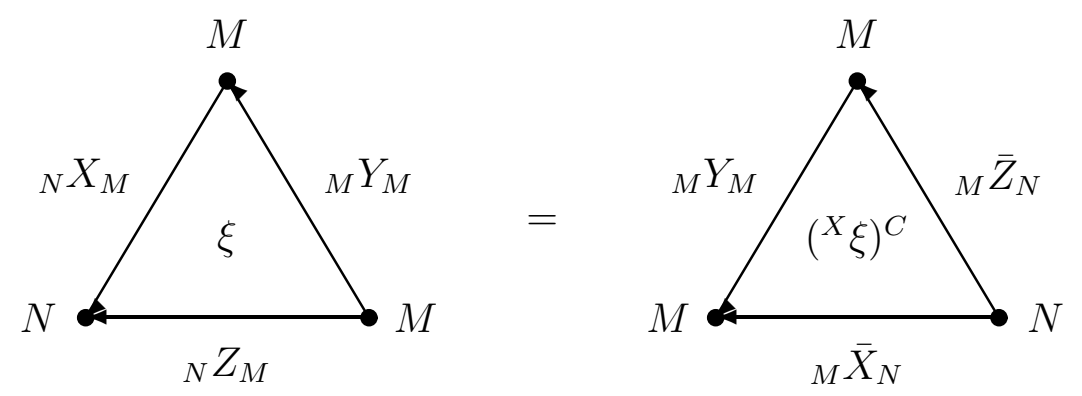

Figure 2.3

We can also take the mirror image of Figure 2.2, and then make the following identification as in Figure 2.4 again using Frobenius reciprocity.
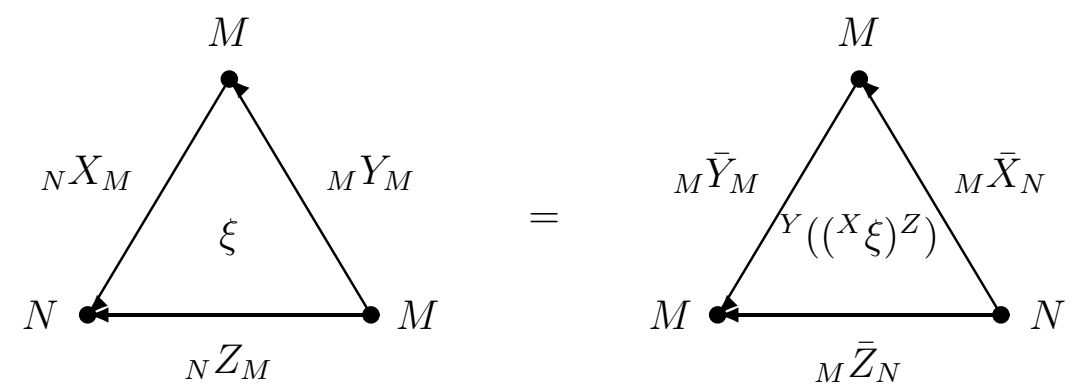

Figure 2.4 
Next assign a finite dimensional Hilbert space to each surface $S$ with a boundary $\partial S$ so that the boundary is divided into oriented edges which are labeled as above. (See [42, Section 2].) Fix a triangulation of $S$ and labeling of its vertices by $N, M$ that are compatible with the division on $\partial S$. Let $H^{\prime}$ be the finite dimensional vector space spanned by all the possible labelings of the triangulated $S$ that are compatible with the labels on $\partial S$. (Because the labeling is given by intertwiners, we have a natural vector space structure.) Let $I S=I \times S / I \times \partial S$, where $I$ is the unit interval $[0,1]$. This is a 3 -manifold with boundary. Two elements in $H^{\prime}$ give a 3 -manifold $I S$ with labeled boundary, so the general partition function scheme gives a number as a topological invariant [9], [11], [29], [42]. This gives a (possibly degenerate) innerproduct on $H^{\prime}$. We define $H_{S}$ to be the quotient of $H^{\prime}$ by the degenerate vectors. Then this space $H_{S}$ is independent of the choice of a triangulation of $S$ as in [42] and its dimension is equal to the value of the topological invariant of the 3-manifold $S \times S^{1}$. (The proof in [42] for the case $\partial S=\varnothing$ works in the same way here.)

Next we modify the path algebra construction in [10], [26], [27], [28] so that the algebra is expressed in terms of two-dimensional pictures instead of one-dimensional pictures. First note that $M^{\mathrm{op}}$ is naturally identified with $M^{\prime} \cap M_{\infty}=\bigcup_{k} \operatorname{End}\left({ }_{M} M_{k M}\right)$. Consider Figure 2.5.

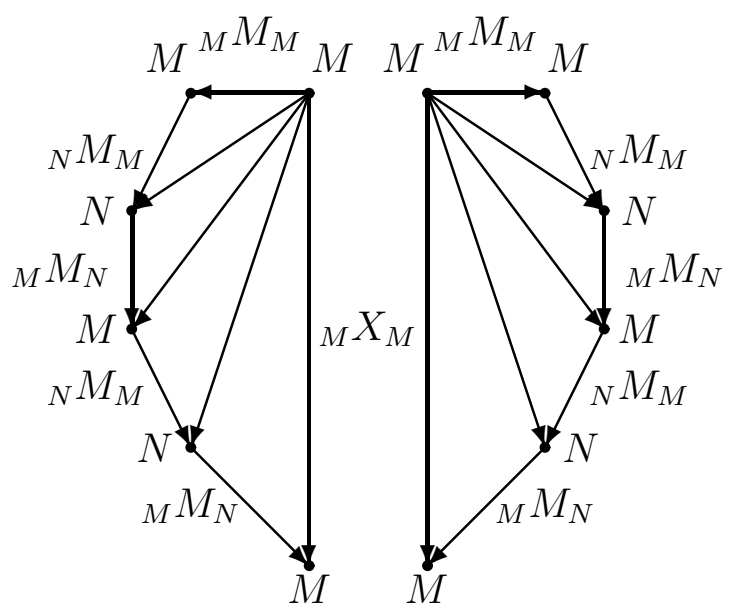

Figure 2.5

Putting labels of intertwiners on Figure 2.5, the left hand side picture gives an intertwiner in $\operatorname{Hom}\left({ }_{M} M \otimes_{N} M \otimes_{M} M \otimes_{N} M \otimes_{M} M_{M},{ }_{M} X_{M}\right)$ and the right hand picture one in $\operatorname{Hom}\left({ }_{M} X_{M},{ }_{M} M \otimes_{N} M \otimes_{M} M \otimes_{N} M \otimes_{M} M_{M}\right)$. Thus by composing these two intertwiners, we get an element in $\operatorname{End}\left({ }_{M} M \otimes_{N} M \otimes_{M} M \otimes_{N} M \otimes_{M} M_{M}\right)=M_{-4}^{\prime} \cap M$. In this way, Figure 2.6, where labeling is dropped for simplicity, ia regarded as an element in $M_{-4}^{\prime} \cap M$. 


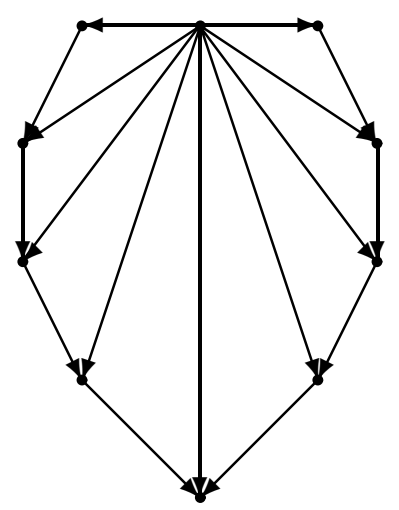

Figure 2.6

Note that in this expression, the embedding from $M_{-4}^{\prime} \cap M$ into $M_{-5}^{\prime} \cap M$ is given by Figure 2.7.
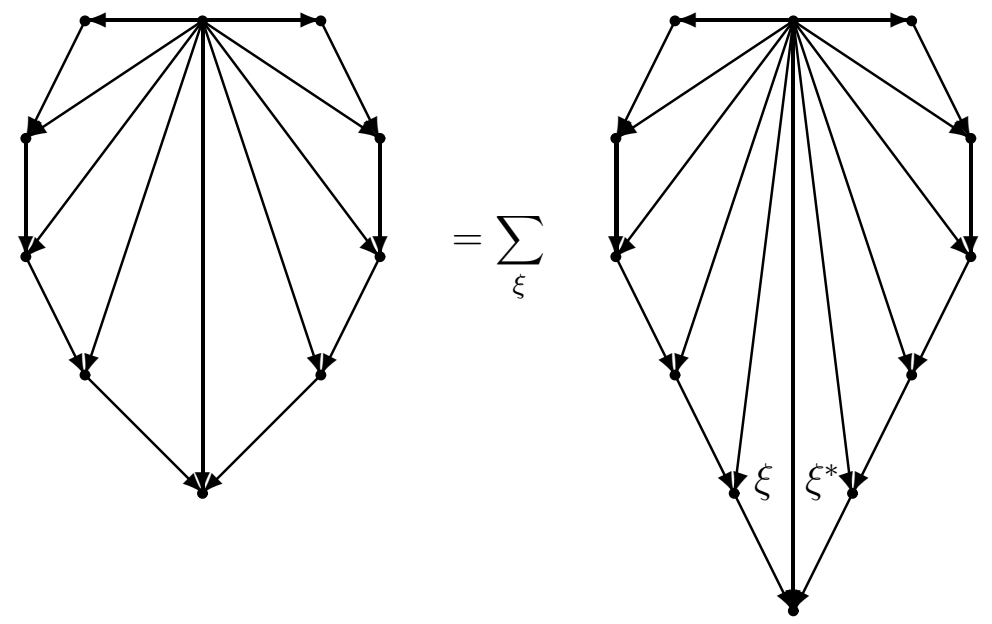

Figure 2.7

(With this embedding, the increasing sequence gives the algebra $M$ instead of $M^{\prime} \cap$ $M_{\infty}$.) In this graphic expression, there is a natural multiplication structure, which is just a rewriting of the multiplication structure of the path algebra.

Next introduce a similar graphic expression of $M-M$ bimodules in the system $\mathcal{M}$. The bimodule is given by an increasing sequence of finite dimensional Hilbert spaces. An element of the finite dimensional Hilbert space is given by Figure 2.8 with labels of intertwiners. 


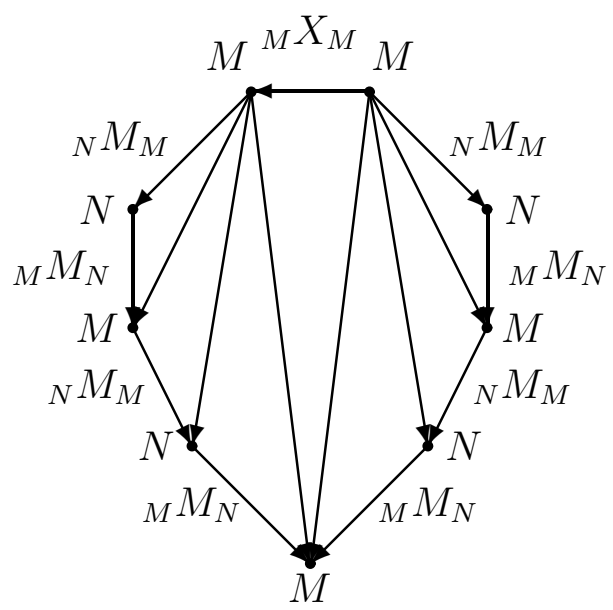

Figure 2.8

The embedding is given by Figure 2.9, where the labeling of vertices/edges is dropped again for simplicity.
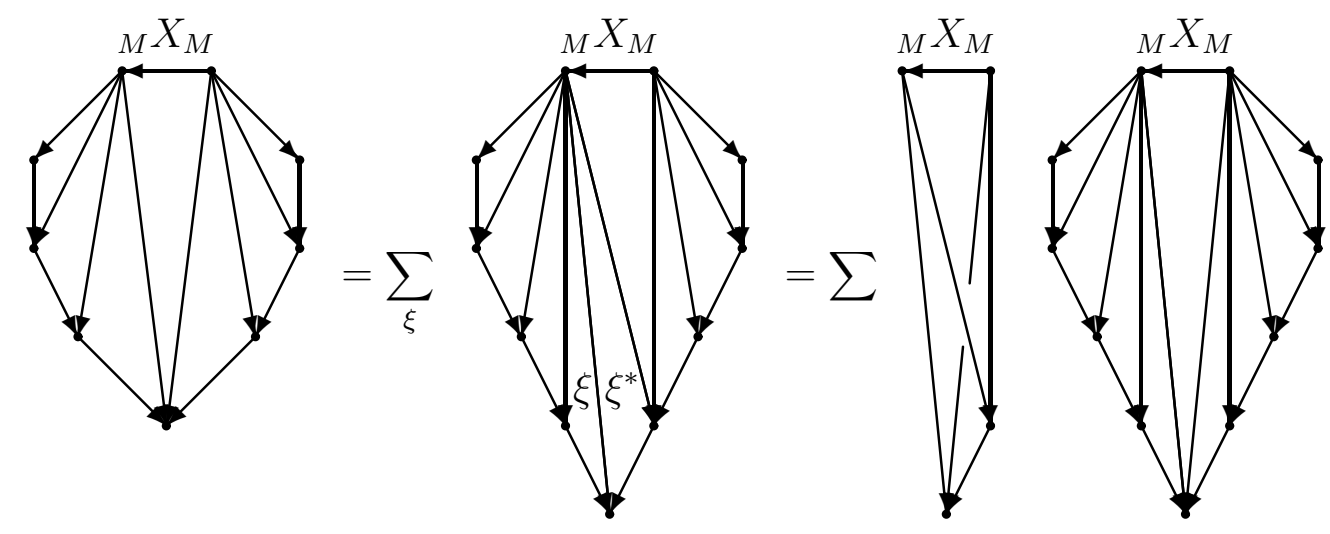

Figure 2.9

Here the coefficient in the right hand side is the (non-normalized) $6 j$-symbol. On each finite dimensional Hilbert space, there is natural left and right actions of $M_{-k}^{\prime} \cap M$, so by taking the completion of the union with respect to a natural inner product, an $M-M$ bimodule is obtained. Call this a surface bimodule and denote this by $A_{X}$.

Note that there is the following basis change rule Figure 2.10 for paths in terms of the connections in the path algebra construction [26], [27], [28].

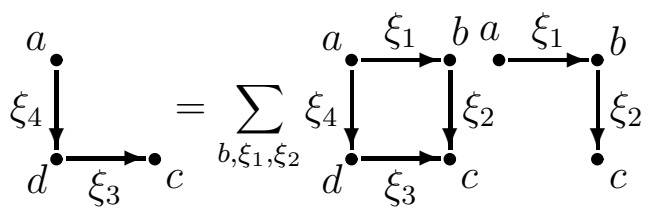

Figure 2.10 
The new basis change rule Figure 2.11 for surfaces in terms of the (non-normalized) $6 j$-symbols is as follows, which is essentially a rewriting of Figure 2.10.
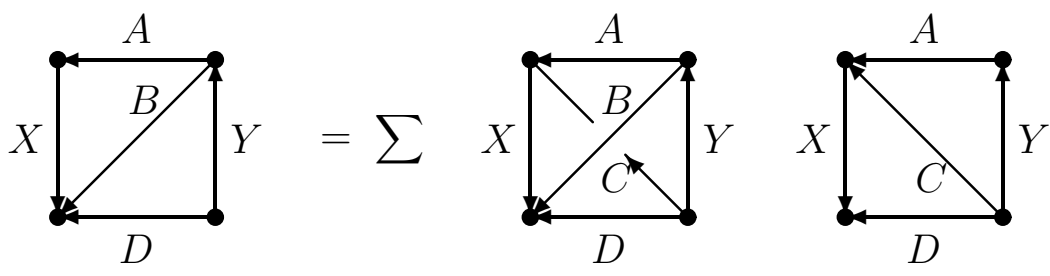

Figure 2.11

These surface bimodules are irreducible.

Theorem 2.1 The bimodule $A_{X}$ is irreducible.

Proof: First, note that there is the following decomposition rule Figure 2.12 of the tensor products of the surface bimodules graphically.

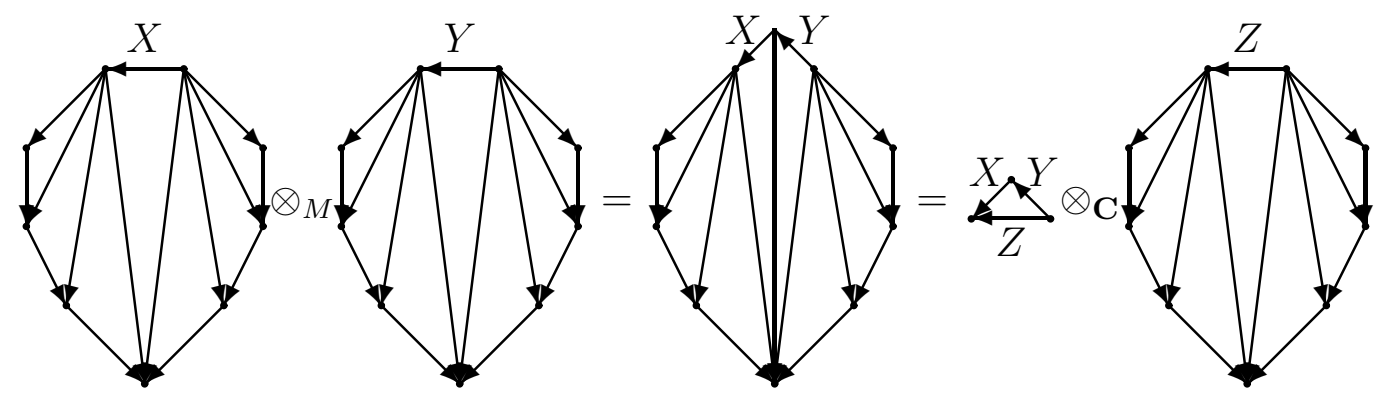

Figure 2.12

Here the triangle on the right hand sides denotes a Hilbert space with dimension $N_{X Y}^{Z}$, the structure constant of the fusion algebra. That is, $A_{X} \otimes_{M} A_{Y}=\sum_{Z} N_{X, Y}^{Z} A_{Z}$. (At this point, it is not known yet that this is the irreducible decomposition.)

Let $\mu_{X}$ be the square root of the Jones index $\left[A_{X}\right]$ corresponding to the bimodule $A_{X}$, that is, the square root of the product of the dimension of $A_{X}$ as a left module and that as a right module. Note that $A_{*}$ for $X=*={ }_{M} M_{M}$ is clearly isomorphic to ${ }_{M} M_{M}$ and hence $\mu_{*}=1$. Because of $\mu_{X} \mu_{Y}=\sum_{Z} N_{X, Y}^{Z} \mu_{Z}, \mu_{*}=1$, and uniqueness of the Perron-Frobenius eigenvector, we get $\mu_{X}=[X]^{1 / 2}$ for each $M-M$ bimodule $X$ in the system $\mathcal{M}$. Let $R_{X}$ be the von Neumann algebra corresponding to Figure 2.13. 


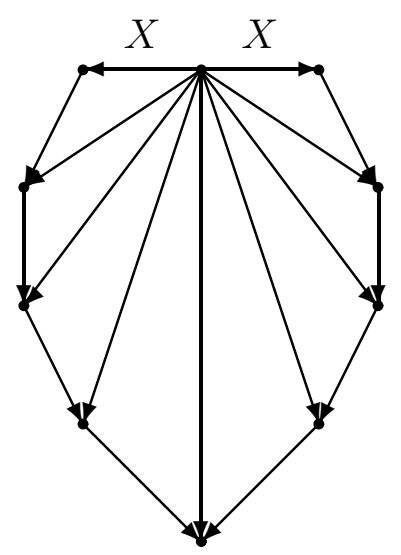

Figure 2.13

(Note that $R_{*}$ is naturally identified with $M$.) Then the bimodule $A_{X}$ has a natural left action of $R_{X}$ in addition to the left and right actions of $M$. There is a natural embedding of $M$ into $R_{X}$ with index $[X]$, and the left action of $R_{X}$ and the right action of $M$ on $A_{X}$ commute. By computing the index, we know that the commutant of the right $M$ action on $A_{X}$ is the left $R_{X}$ action. The natural embedding of $M$ into $R_{X}$ has a trivial relative commutant by Wenzl's dimension estimate [45, Theorem 1.6] (or Ocneanu's compactness argument [28, II.6]), hence we know that the bimodule $A_{X}$ is irreducible.

Q.E.D.

With this irreducibility and the decomposition rule of the tensor products, we can identify the systems of surface bimodules with the original system $\mathcal{M}$ of bimodules.

\section{Tube algebra, the Hilbert space $H_{S^{1} \times S^{1}}$, and la- beling for circles}

In the study of the Hilbert spaces associated to surfaces with labeled boundaries as above, we encounter the problem to label circles which are parts of boundaries of surfaces in more natural way. For this purpose, a new algebra, the tube algebra, as in [30], [31], [34], [35], is introduced and it is shown that its center gives a natural labeling of circles in the TQFT. This section is based on [31], [32], [33], [34].

Take $M$ - $M$ bimodules $X, X^{\prime}, Y, Y^{\prime}, A, A^{\prime}, B$ in the system $\mathcal{M}$. We define a finite dimensional $C^{*}$-algebra Tube $\mathcal{M}$

$$
\text { Tube } \mathcal{M}=\oplus_{X, Y, A} \operatorname{Hom}(X \otimes A, A \otimes Y)
$$

with the product defined by

$$
\xi \cdot \xi^{\prime}=\delta_{Y, X^{\prime}} \sum_{B, \alpha}\left(\alpha \otimes 1_{Y^{\prime}}\right) \cdot\left(1_{A} \otimes \xi^{\prime}\right) \cdot\left(\xi \otimes 1_{A^{\prime}}\right) \cdot\left(1_{X} \otimes \alpha^{*}\right)
$$


where $\xi \in \operatorname{Hom}(X \otimes A, A \otimes Y), \xi^{\prime} \in \operatorname{Hom}\left(X^{\prime} \otimes A^{\prime}, A^{\prime} \otimes Y^{\prime}\right)$ and $\alpha$ is in a basis of $\operatorname{Hom}\left(A \otimes A^{\prime}, B\right)$. Graphically, an element in this algebra and the product is expressed as in Figure 3.1.

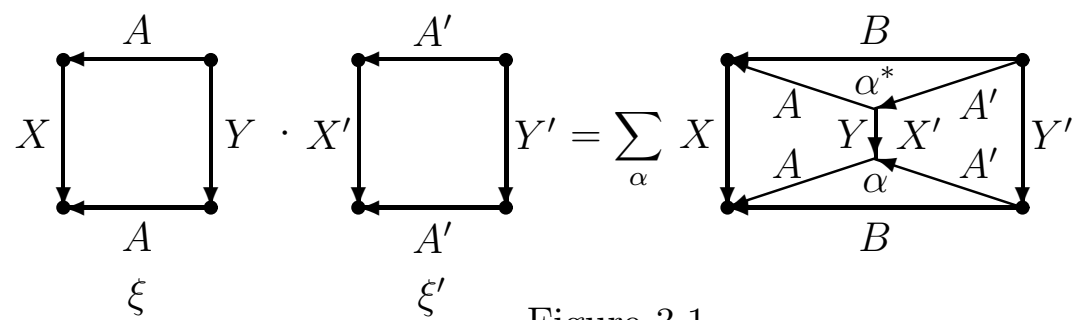

Figure 3.1

In the expression of $\xi$ in Figure 3.1, the top and the bottom edges are labeled by the same bimodule $A$, and the square is regarded as a tube with this identification. The name tube algebra comes from this. (See Figure 3.2.)

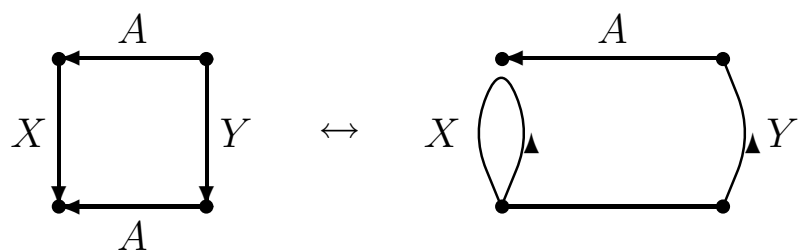

Figure 3.2

In the Turaev-Viro type TQFT arising from the system $\mathcal{M}$ as in [11], [29], we study the Hilbert space $H_{S^{1} \times S^{1}}$ associated to the 2-dimensional torus $S^{1} \times S^{1}$, which is defined as in Section 2. Note that the topological invariant of the 3-dimensional torus in this TQFT gives the dimension of this Hilbert space. For the study of this space, we can fix any triangulation of the 2-dimensional torus, so we choose the one as in Figure 3.3.

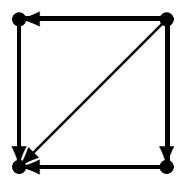

Figure 3.3

In this picture, the top and the bottom edges are identified, and the left and right edges are identified to give a torus. This is not a triangulation in the standard terminology in combinatorial topology, because it has only one vertex, but this does 
not matter for our purpose. To compute the Hilbert space $H_{S^{1} \times S^{1}}$, assign an algebra $M$ to any vertex so that only $M-M$ bimodules in $\mathcal{M}$ are used. If $\xi \in$ Tube $\mathcal{M}$ with the above graphical expression is in the center of Tube $\mathcal{M}$, it is easy to see that we have $X=Y$, so an element in the center of Tube $M$ naturally gives an element in $H_{S^{1} \times S^{1}}$ as the same intertwiner on the same picture in $H_{S^{1} \times S^{1}}^{\prime}$ modulo the degenerate vectors. Ocneanu's claim in [34] is that this gives an isomorphism.

Theorem 3.1 The center of the tube algebra $\mathcal{M}$ is identified with the Hilbert space $H_{S^{1} \times S^{1}}$ associated to the TQFT arising from $\mathcal{M}$.

Proof: This is proved graphically as follows. These pictures are taken from [33, Figure 8.5], where Ocneanu works on the two-dimensional theory rather than the three-dimensional theory. Because it is easier to draw two-dimensional pictures than three-dimensional pictures, we have Figures 3.4 and 3.5 with dimension one less as in [33]. To get the real pictures for the current situation, it is enough to multiply everything below with $S^{1}$.

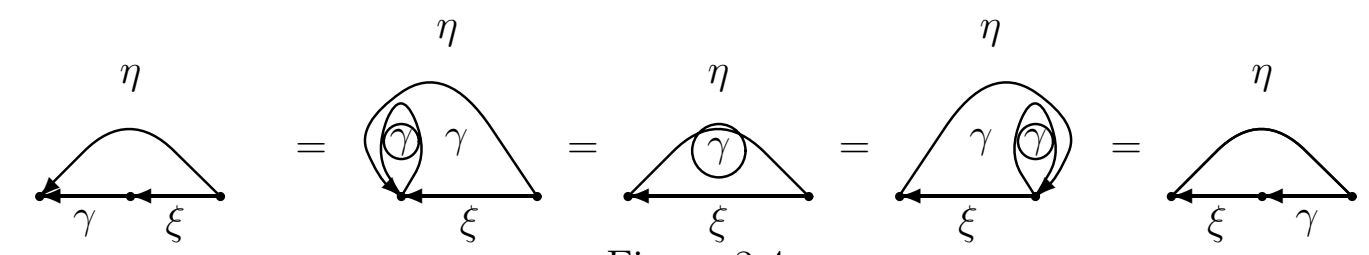

Figure 3.4

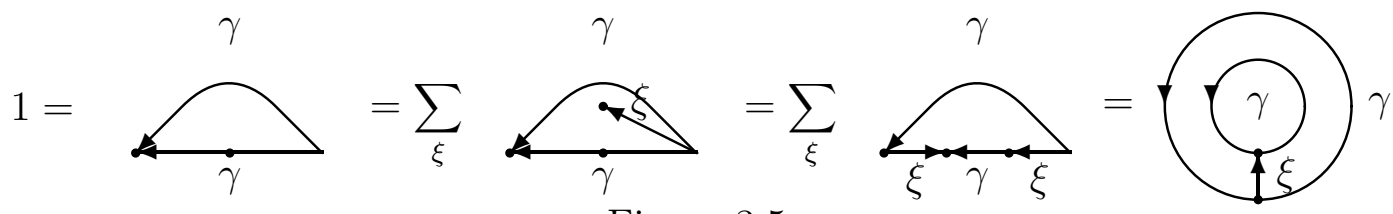

Figure 3.5

The interiors of the small inner disks labeled with $\gamma$ in the second, third, and fourth pictures of Figure 3.4 and in the fourth picture of Figure 3.5 are all empty.

Figure 3.4 shows that an element $\gamma$ in $H_{S^{1} \times S^{1}}$ commutes with any $\xi$ in the tube algebra. Figure 3.5 shows that a central element $\gamma$ in the tube algebra is in $H_{S^{1} \times S^{1}}$.

Q.E.D.

Denote the set of the minimal central projections of Tube $\mathcal{M}$ by $\left\{\pi_{1}, \cdots, \pi_{n}\right\}$. Label circles, which are parts of boundaries of surfaces, with $\pi_{j}$ 's.

First rewrite the picture of $\pi_{j}$ as in Figure 3.6. 


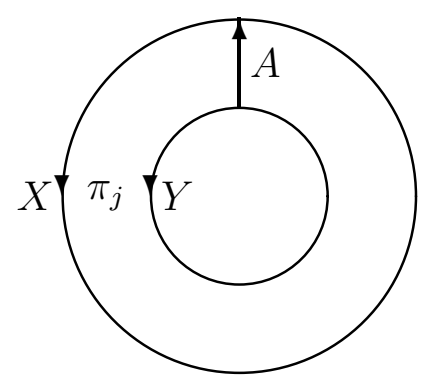

Figure 3.6

(Actually, there is a labeling for the annulus with intertwiners and a summation over $A, X, Y$, which we have dropped for simplicity.)

By labeling a circle, which is a part of the boundary of a surface $S$, with $\pi_{j}$, we mean the following. Thicken the circle so that there is an annulus which has the circle as a common boundary with $S$. Then we have a new surface, a part of which is labeled with intertwiners. We can still assign a Hilbert space to this partially labeled surface just as in Section 2. Then the product rule $\pi_{j} \pi_{k}=\delta_{j k} \pi_{j}$ and the minimality of $\pi_{j}$ imply that the dimension of the Hilbert space for the annulus in Figure 3.7 is $\delta_{j k}$.

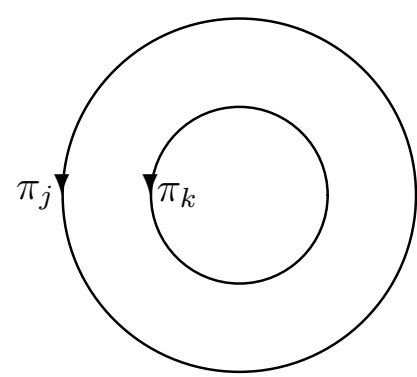

Figure 3.7

\section{Tensor products of bimodules for the asymp- totic inclusion}

The contents the previous section were mostly combinatorial. The aim in this section is to give them an operator algebraic meaning, as sketched in [34]. That is, the "convolution product" in the center of the tube algebra will be identified with the fusion rule of $M_{\infty}-M_{\infty}$ bimodules of the asymptotic inclusion. In particular, it will then turn out that the tensor product operation of the $M_{\infty}-M_{\infty}$ bimodules arising from the asymptotic inclusion is commutative, as claimed in [35]. This is not trivial at all from the definition of the asymptotic inclusion.

We first introduce a new commutative multiplication $*$, a convolution, on $H_{S^{1} \times S^{1}}$ as follows, as in [31]. Let $N_{i j}^{k}$ be the dimension of the Hilbert space associated to 
the partially labeled surface in Figure 4.1. (Here this picture is a disk with two disks removed and has three boundary components.)

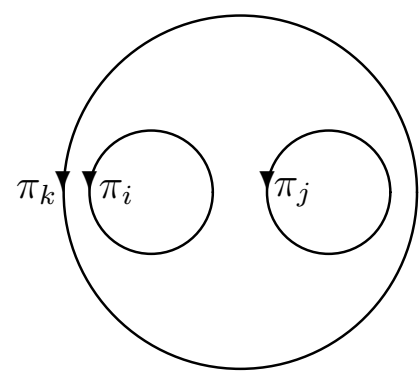

Figure 4.1

We define $\pi_{i} * \pi_{j}=\sum_{k} N_{i j}^{k} \pi_{k}$ and extend this linearly to $H_{S^{1} \times S^{1}}$. The commutativity follows from that switching $\pi_{i}$ and $\pi_{j}$ in Figure 4.1 gives a homeomorphic picture. We call the algebra $H_{S^{1} \times S^{1}}$ with this convolution product the asymptotic fusion algebra of the original system $\mathcal{M}$ of bimodules.

In order to deal with bimodules arising from the asymptotic inclusion $M \vee\left(M^{\prime} \cap\right.$ $\left.M_{\infty}\right) \subset M_{\infty}$, we first express the algebras $M \vee\left(M^{\prime} \cap M_{\infty}\right)$ and $M_{\infty}$ graphically. Because $M^{\prime} \cap M_{\infty}$ is naturally identified with $M^{\mathrm{op}}$, we express the algebra $M \vee\left(M^{\prime} \cap\right.$ $\left.M_{\infty}\right)$ with Figure 4.2 .
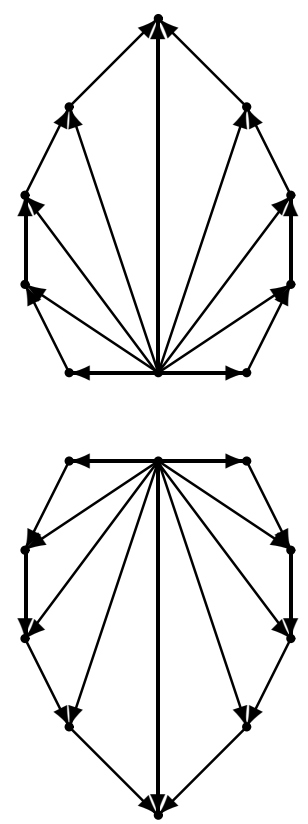

Figure 4.2

Here the bottom copy for $M$ is the same as in Section 2 and the top copy is for $M^{\text {op }}$. We have the same embedding rule as in Section 2. The algebra $M_{\infty}$ is expressed as $\bigvee_{j, k}\left(M_{-j}^{\prime} \cap M_{k}\right)$ for a generating tunnel $\left\{M_{-j}\right\}_{j}$, so we have Figure 4.3. 


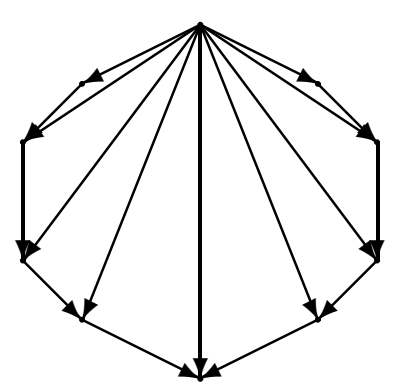

Figure 4.3

The upward and downward embeddings are expressed as in Figure 4.4.

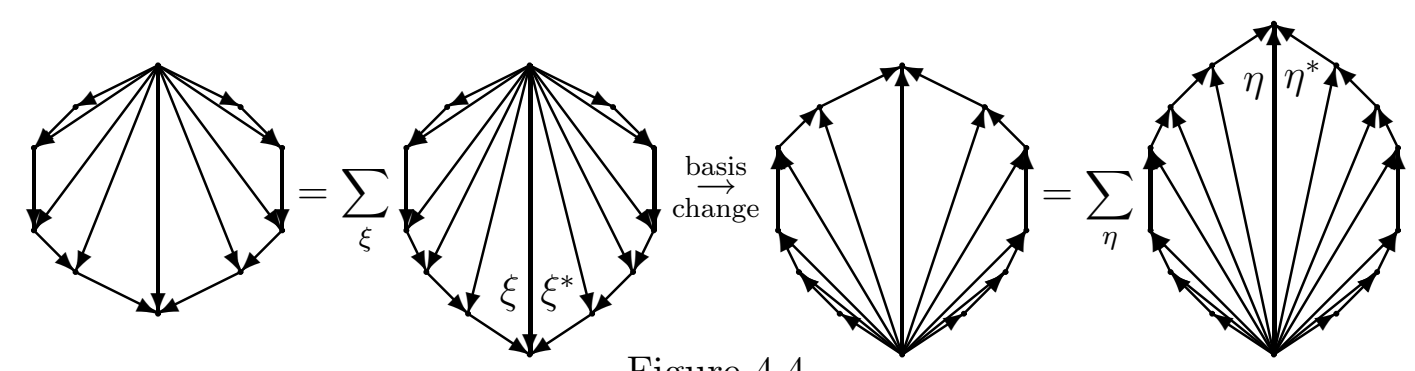

Figure 4.4

Next the algebra $M_{\infty}$ as an $M \vee\left(M^{\prime} \cap M_{\infty}\right)-M_{\infty}$ bimodule is expressed with the following picture. Again this has two directions of embeddings as in Figure 4.5.

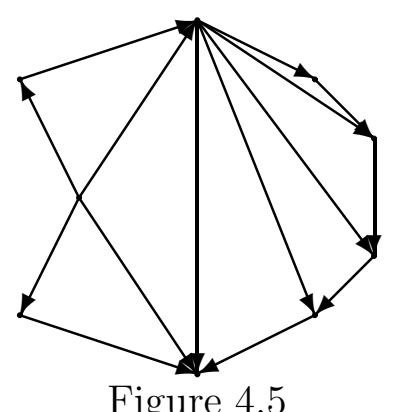

With the above preliminaries, we now deal with bimodules arising from the asymptotic inclusion $M \vee\left(M^{\prime} \cap M_{\infty}\right) \subset M_{\infty}$. First, we handle $M \vee\left(M^{\prime} \cap M_{\infty}\right)-M \vee\left(M^{\prime} \cap M_{\infty}\right)$ bimodules. They are expressed with Figure 4.6. 


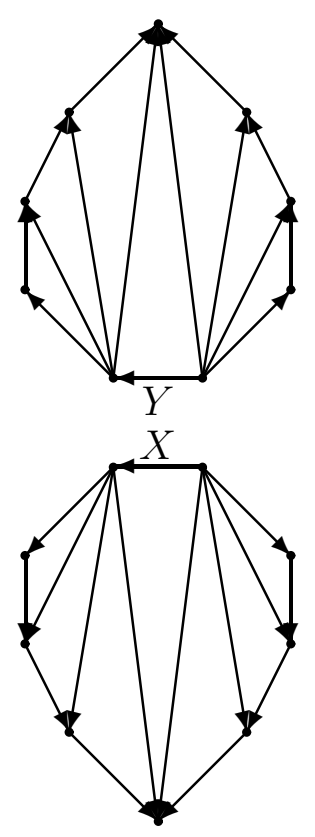

Figure 4.6

where $X$ and $Y$ are any pair of $M-M$ bimodules of the original system $\mathcal{M}$ and this picture again has two directions of embeddings. We denote this bimodule by $B_{X, Y}$. This is essentially just a pair of $M-M$ bimodules, and the tensor product structure is easy to see. In particular, this bimodule is irreducible as an $M \vee\left(M^{\prime} \cap M_{\infty}\right)$ $M \vee\left(M^{\prime} \cap M_{\infty}\right)$ bimodule.

Next we multiply the $M \vee\left(M^{\prime} \cap M_{\infty}\right)-M \vee\left(M^{\prime} \cap M_{\infty}\right)$ bimodule in Figure 4.6 with $M \vee\left(M^{\prime} \cap M_{\infty}\right) M_{\infty M_{\infty}}$ from the right. Then we have the $M \vee\left(M^{\prime} \cap M_{\infty}\right)-M_{\infty}$ bimodule as in Figure 4.7.

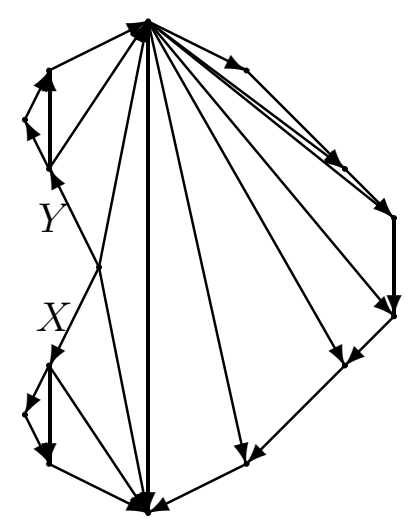

Figure 4.7

It is decomposed as in Figure 4.8. 


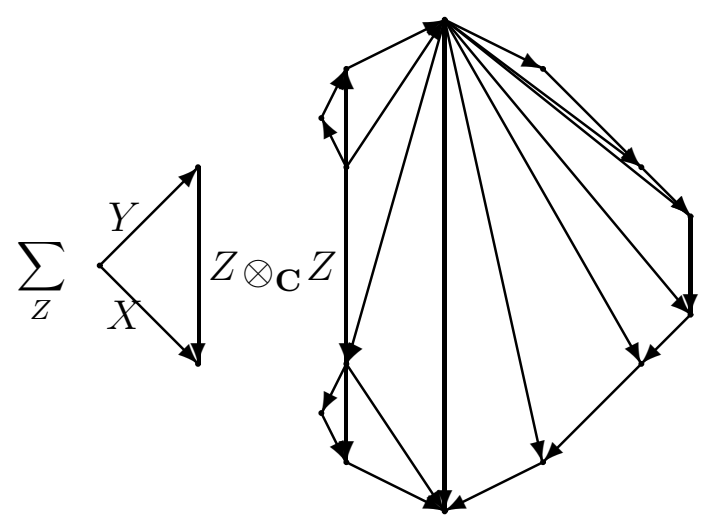

Figure 4.8

It is claimed that this is really an irreducible decomposition. Note that the dimension of the Hilbert space associated to the triangle on the right hand side is $N_{X, \bar{Y}}^{Z}$.

Theorem 4.1 An $M \vee\left(M^{\prime} \cap M_{\infty}\right)-M_{\infty}$ bimodule given by Figure 4.9 is irreducible.

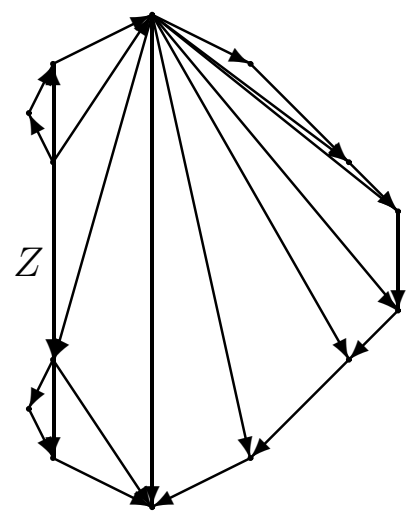

Figure 4.9

Proof: We denote the bimodule in Figure 4.9 by $B_{Z}$ for an $M-M$ bimodule $Z$ in $\mathcal{M}$. Note that $B_{*}={ }_{M \vee\left(M^{\prime} \cap M_{\infty}\right)} M_{\infty M_{\infty}}$. Then a graphical inspection easily produces

$$
B_{Z} \otimes_{M_{\infty}} M_{\infty M \vee\left(M^{\prime} \cap M_{\infty}\right)}=\bigoplus_{X, Y} N_{X, \bar{Y}}^{Z} B_{X, Y}
$$

which is the irreducible decomposition.

Let $B_{j}$ 's be the irreducible $M \vee\left(M^{\prime} \cap M_{\infty}\right)-M_{\infty}$ bimodules in the system. Suppose we have $B_{Z}=\oplus_{j} n_{j}^{Z} B_{j}$. Suppose we have $B_{X, Y} \otimes_{M \vee\left(M^{\prime} \cap M_{\infty}\right)} M_{\infty M_{\infty}}=\sum_{j} n_{j}^{X, Y}$ as an irreducible decomposition. Then the above irreducible decomposition of $B_{Z} \otimes_{M_{\infty}}$ $M_{\infty M \vee\left(M^{\prime} \cap M_{\infty}\right)}$ and the formula $B_{X, Y} \otimes_{M \vee\left(M^{\prime} \cap M_{\infty}\right)} M_{\infty M_{\infty}}=\sum_{j} n_{j}^{X, Y}$ with Frobenius reciprocity give $N_{X, \bar{Y}}^{Z}=\sum_{j} n_{j}^{Z} n_{j}^{X, Y}$. We set $\mu_{j}=\left[B_{j}\right]^{1 / 2}$ and $\mu_{Z}=\left[B_{Z}\right]^{1 / 2}$. Then Figure 4.8 shows

$$
\sum_{j} n_{j}^{X, Y} \mu_{j}=\left[B_{X, Y}\right]^{1 / 2}\left[{ }_{M \vee\left(M^{\prime} \cap M_{\infty}\right)} M_{\infty} M_{\infty}\right]^{1 / 2}=\sum_{Z} N_{X, \bar{Y}}^{Z} \mu_{Z}
$$


We also have

$$
\sum_{Z} N_{X, \bar{Y}}^{Z} \mu_{Z}=\sum_{Z, j, k}\left(n_{k}^{Z} n_{k}^{X, Y}\right)\left(n_{j}^{Z} \mu_{j}\right)=\sum_{j}\left(\sum_{Z, k} n_{k}^{Z} n_{k}^{X, Y} n_{j}^{Z}\right) \mu_{j} \geq \sum_{j}\left(\sum_{Z}\left(n_{j}^{Z}\right)^{2} n_{j}^{X, Y}\right) \mu_{j} .
$$

We fix $X, Y$. Note that for any $j$, we have $Z$ with $n_{j}^{Z} \geq 1$. We thus get

$$
\sum_{Z}\left(n_{j}^{Z}\right)^{2} n_{j}^{X, Y}=n_{j}^{X, Y}
$$

for all $j$.

We next fix $j$ and choose $X, Y$ so that $n_{j}^{X, Y} \geq 1$. Then we have $\sum_{Z}\left(n_{j}^{Z}\right)^{2}=1$, that is, for any $j$ we have only one $Z$ with $n_{j}^{Z} \geq 1$ and we have $n_{j}^{Z}=1$ for this $Z$.

We next fix $Z$ and choose $j, X, Y$ with $n_{j}^{Z} \geq 1$ and $N_{X, \bar{Y}}^{Z} \geq 1$. These imply $n_{j}^{X, Y} \geq 1$. Then we have

$$
n_{j}^{X, Y}=\sum_{k, Z^{\prime}} n_{k}^{Z^{\prime}} n_{k}^{X, Y} n_{j}^{Z^{\prime}}=\left(\sum_{k} n_{k}^{Z} n_{k}^{X, Y}\right) n_{j}^{Z}
$$

This implies $n_{k}^{Z} n_{k}^{X, Y}=0$ for all $k \neq j$. This implies the irreducible decomposition of $B_{Z}$ has just one summand $B_{j}$. This means each $B_{Z}$ is irreducible.

Q.E.D.

This describes the principal graph of the asymptotic inclusion as follows. We have a system of $M \vee\left(M^{\prime} \cap M_{\infty}\right)-M \vee\left(M^{\prime} \cap M_{\infty}\right)$ bimodules $B_{X, Y}$ labeled by $X, Y$ in $\mathcal{M}$ and a system of $M \vee\left(M^{\prime} \cap M_{\infty}\right)-M_{\infty}$ bimodules $B_{Z}$ labeled by $Z$ in $\mathcal{M}$. We have the fusion graph whose vertices are bimodules $B_{X, Y}$ and $B_{Z}$ and which has $N_{X, \bar{Y}}^{Z}$ edges between $B_{X, Y}$ and $B_{Z}$. The connected component of the fusion graph containing the vertex $*$ is the principal graph of the subfactor $M \vee\left(M^{\prime} \cap M_{\infty}\right) \subset M_{\infty}$, where $*$ denotes the identity bimodule ${ }_{M} M_{M}$. This proves a theorem claimed in $[28$, III.1] without a proof and also left without proof in [18, Remark 2.16]. That is, the connection for the natural commuting square

$$
\begin{array}{cccc}
\left(M_{-k}^{\prime} \cap M_{0}\right) \vee\left(M_{0}^{\prime} \cap M_{k}\right) & \subset & \left(M_{-k-1}^{\prime} \cap M_{0}\right) \vee\left(M_{0}^{\prime} \cap M_{k+1}\right) \\
M_{-k}^{\prime} \cap M_{k} & \subset & M_{-k-1}^{\prime} \cap M_{k+1}
\end{array}
$$

is flat. The other statement of Ocneanu [28] left unproved in [18, Remark 2.16] is proved in [21, Appendix].

Next we have to deal with $M_{\infty}-M_{\infty}$ bimodules. First we have a graphical expression for an $M_{\infty}-M \vee\left(M^{\prime} \cap M_{\infty}\right)$ bimodule $\bar{B}_{Z}$ as in Figure 4.10. 


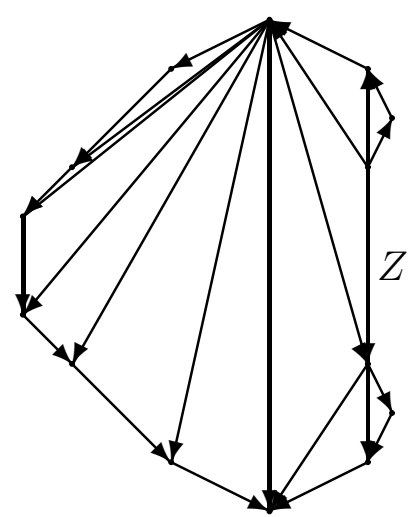

Figure 4.10

We make a tensor product $\bar{B}_{Z} \otimes_{M \vee\left(M^{\prime} \cap M_{\infty}\right)} B_{Z^{\prime}}$. First we have a graphical expression as in Figure 4.11.

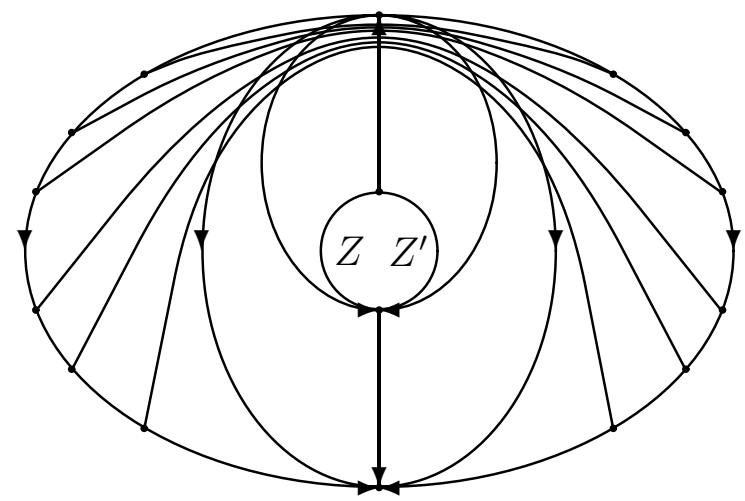

Figure 4.11

Here the interior of the inner disk is empty. We need an irreducible decomposition of this $M_{\infty}-M_{\infty}$ bimodule. Denote by $N_{Z, Z^{\prime}}^{\pi_{j}}$ the dimension of the Hilbert space associated to the annulus in Figure 4.12.

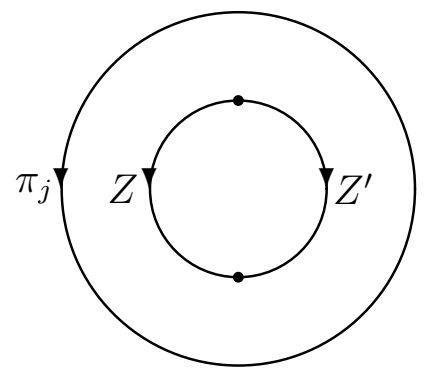

Figure 4.12

Here the boundary of this annulus consists of two circles. The inner circle has two components and they are labeled by $Z$ and $Z^{\prime}$ respectively. The outer circle is labeled 
by $\pi_{j}$. Because $\pi_{j}$ is a projection in the tube algebra, we get the decomposition of the $M_{\infty}-M_{\infty}$ bimodule as in Figure 4.13.

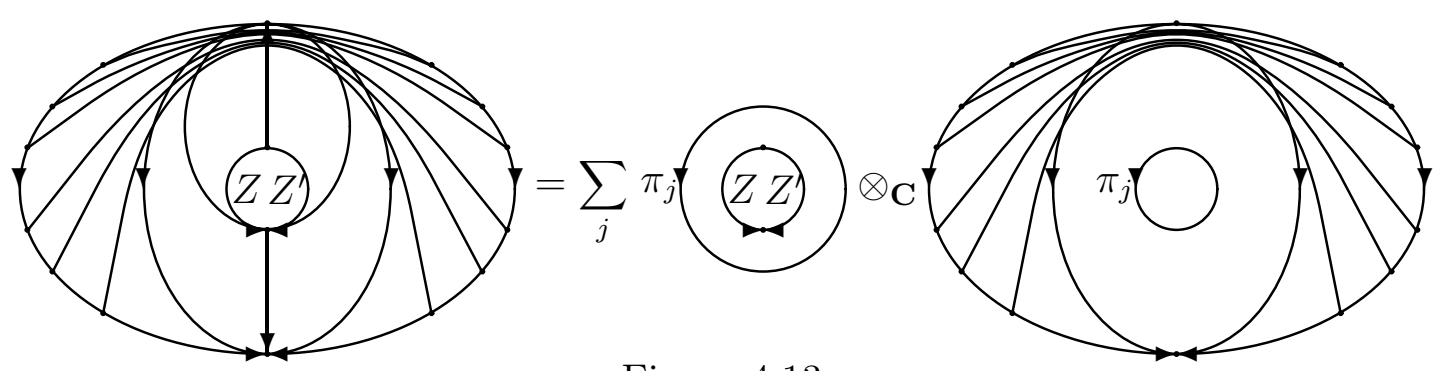

Figure 4.13

Denote this $M_{\infty}-M_{\infty}$ bimodule on the right hand by $B_{\pi_{j}}$. It is then claimed that the decomposition in Figure 4.13 is the irreducible decomposition.

Theorem 4.2 Each $B_{\pi_{j}}$ is an irreducible $M_{\infty}-M_{\infty}$ bimodule.

Proof: A graphical expression yields the identity

$$
B_{\pi_{j}} \otimes_{M_{\infty}} M_{\infty M \vee\left(M^{\prime} \cap M_{\infty}\right)}=\bigoplus_{Z} N_{Z, *}^{\pi_{j}} B_{Z},
$$

which is the irreducible decomposition as an $M_{\infty}-M \vee\left(M^{\prime} \cap M_{\infty}\right)$ bimodule. Then the same argument as in the proof of Theorem 3.1 works.

It is easy to see that the system of bimodules $B_{\pi_{j}}$ is closed under the tensor product of $M_{\infty}-M_{\infty}$ bimodules. Call this system the tube system and denote it by $\mathcal{M}_{\infty}$. The tensor product in the tube system is given by the following.

Theorem 4.3 In $\mathcal{M}_{\infty}$, we have

$$
B_{\pi_{i}} \otimes_{M_{\infty}} B_{\pi_{j}}=\bigoplus_{k} N_{i j}^{k} B_{\pi_{k}}
$$

Proof: $\quad$ The bimodule $B_{\pi_{i}} \otimes_{M_{\infty}} B_{\pi_{j}}$ is expressed as in Figure 4.14.

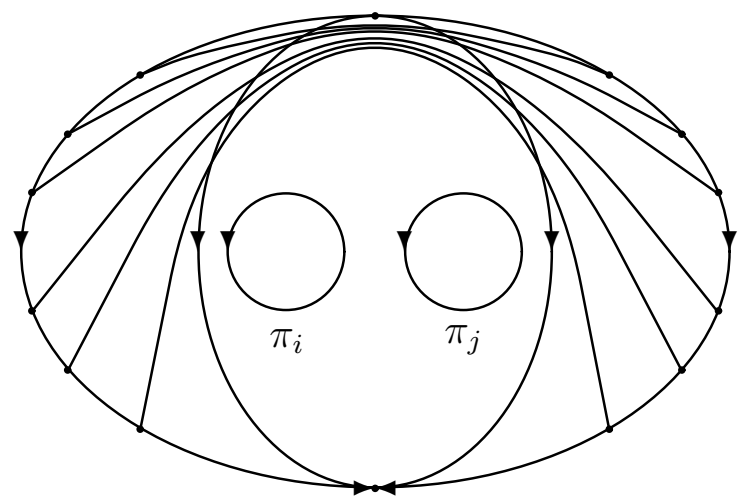

Figure 4.14 

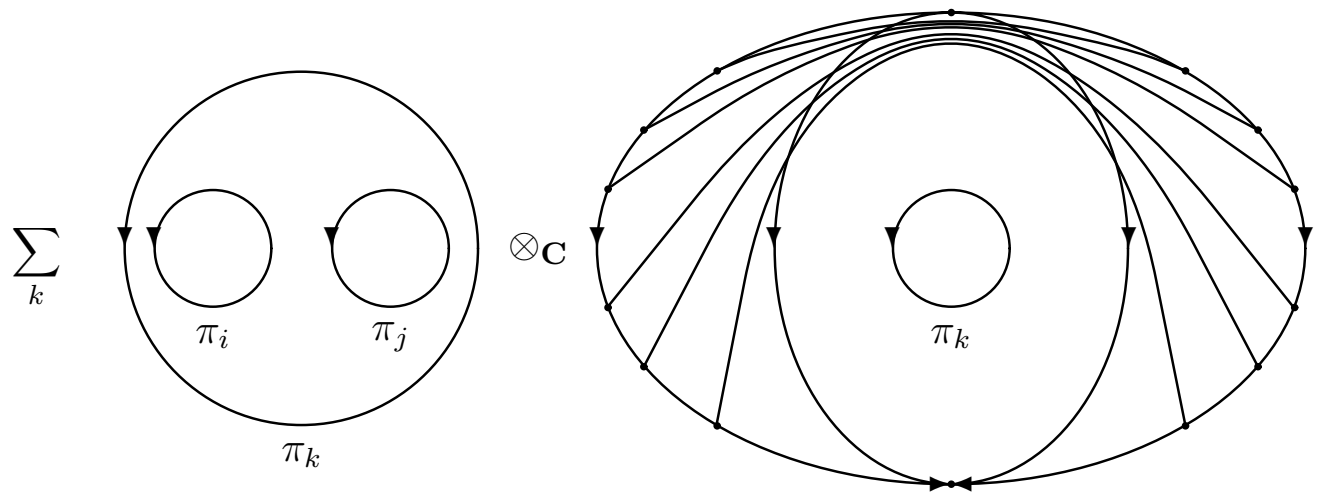

Figure 4.15

Q.E.D.

In this way, the tube system is identified with the asymptotic fusion algebra.

We now have a graph with vertices labeled by $B_{Z}$ and $B_{\pi_{j}}$ with $N_{Z, *}^{\pi_{j}}$ edges between vertices represented by $B_{Z}$ and $B_{\pi_{j}}$. The above theorem shows that the connected component of this graph containing $B_{*}$ is the dual principal graph of the asymptotic inclusion $M \vee\left(M^{\prime} \cap M_{\infty}\right) \subset M_{\infty}$. If the fusion graph is connected, then this graph is also connected. In particular, the above theorem shows that the tensor product operation of the $M_{\infty}-M_{\infty}$ bimodules arising from the asymptotic inclusion is commutative, as mentioned above.

Thus if the fusion graph is connected, there is an identification between the natural basis of the Hilbert space $H_{S^{1} \times S^{1}}$ of the TQFT arising from the original subfactor $N \subset M$, the minimal central projections in the tube algebra Tube $\mathcal{M}$ of the original subfactor $N \subset M$, and the irreducible $M_{\infty}-M_{\infty}$ bimodules arising from the asymptotic inclusion $M \vee\left(M^{\prime} \cap M_{\infty}\right) \subset M_{\infty}$. If the fusion graph is not connected, some bimodules in the tube system are not given by the basic constructions from the asymptotic inclusion.

\section{$5 \quad S$-matrix, T-matrix, and the Verlinde identity}

In [3], de Boer and Goeree constructed paragroups from combinatorial data of RCFT satisfying the Moore-Seiberg axioms [25]. In the converse direction, they attempted to construct combinatorial data of RCFT from paragroups. In particular, they tried to construct $S$-matrices from paragroups of type $D$ and $E$ in the last section of [3] without success. (See [2], [13], [16] for paragroups of type $D$ and $E$.) It will be shown that such data can be constructed after passing to the asymptotic inclusion from a given paragroup. That is, the $S$-matrix and $T$-matrix are introduced for the tube system $\mathcal{M}_{\infty}$, and several analogues of the theorems in RCFT [43], [44] are then proved. That is, the $S$-matrix and the $T$-matrix are defined so that the $S$-matrix diagonalizes the fusion algebra of the $M_{\infty}-M_{\infty}$ bimodules of the asymptotic inclusion, 
the $T$-matrix is diagonal, and its diagonal entries are roots of unity. This section is mainly based on Ocneanu's lectures at Collège de France [31].

Define the operations $S, T$ on $H_{S^{1} \times S^{1}}$ graphically as in Figures 5.1 and 5.2.

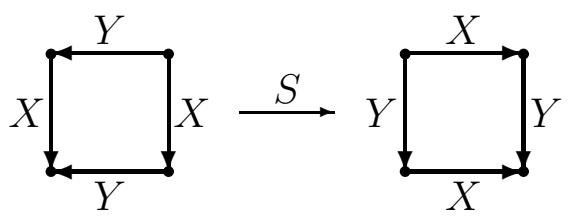

Figure 5.1

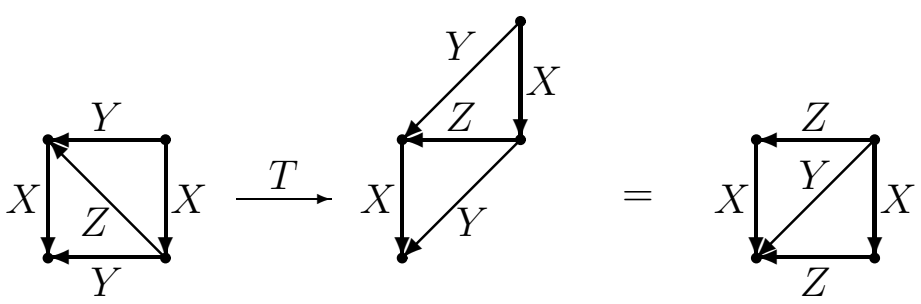

Figure 5.2

It is easy to see that these two operators are unitary on $H_{S^{1} \times S^{1}}$. Note that the operation $T$ means a "twist" of a tube.

It is again easy to see graphically that the $S$-matrix interchanges the two products, the tube algebra product and the convolution product as in Figure 5.3.

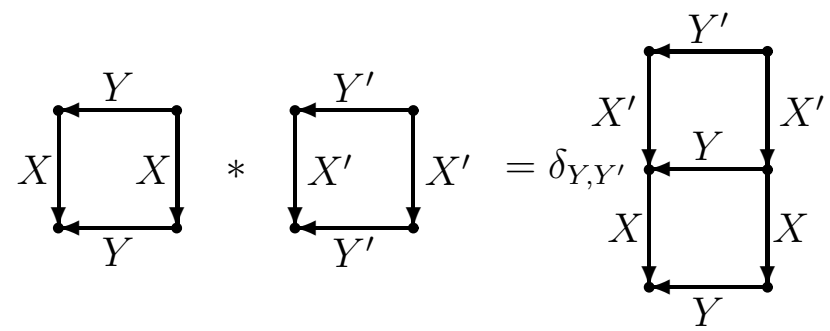

Figure 5.3

Note that in the right hand side, the product is expressed as in Figure 3.1. That is, we have the following theorem.

Theorem 5.1 For $\xi, \eta \in H_{S^{1} \times S^{1}}$, we have

$$
S \xi * S \eta=S(\xi \cdot \eta),
$$

where the product - denotes the multiplication in the tube algebra. That is, the Smatrix diagonalizes the asymptotic fusion algebra. 
This is an analogue of the celebrated theorem of E. Verlinde [44].

Again from Figure 5.4, we have the following identity.

$$
\left(T \xi \cdot T^{*} \eta, \zeta\right)=(\xi \cdot \eta, \zeta), \quad \text { for all } \zeta
$$



Figure 5.4

Thus we have

$$
T \xi \cdot T^{*} \eta=\xi \cdot \eta
$$

which implies the matrix $T$ is diagonal to the natural basis $\pi_{j}$ of the Hilbert space $\pi_{j}$. That is, we have a scalar $t_{j} \in \mathbf{C}$ with $T \pi_{j}=t_{j} \pi_{j}$. Then exactly as in [43], we can prove that the diagonal entries of the $T$-matrix are roots of unity. This was claimed in Ocneanu's conference talk [32]. (Here we need to appeal to Remark \# 7 in [43, page 424].)

In this way, we can also construct data for rational conformal field theory in the combinatorial sense of Moore-Seiberg [25].

See [4] for an example of $S$ - and $T$-matrices. The examples in [30] are the same as these.

\section{Quantum double construction for paragroups}

In this last section, it is explained why this passage from a paragroup to the new paragroup of the asymptotic inclusion can be regarded as a paragroup analogue of the quantum double construction of Drinfel'd [8], as claimed in [34], [35].

We look at the group case in detail, so start with a finite group $G$. Consider the subfactor $N \subset M=N \times G$, where $N$ is the AFD $\mathrm{II}_{1}$ factor and the finite group $G$ acts on $N$ freely. Then the central sequence subfactor $N^{\omega} \cap M^{\prime} \subset M_{\omega}$ is of the form $Q \times G \in Q \times(G \times G)$, where $Q$ is some factor of type $\mathrm{II}_{1}$ with an outer action of $G \times G$ and $G$ is embedded into $G \times G$ with a map $g \mapsto(g, g)$. (This was first claimed in [28, III.3]. See [21, Appendix] for a complete proof.) Then we know that the asymptotic inclusion is of the form $R^{G \times G} \subset R^{G}$, where $R$ is the AFD $\mathrm{II}_{1}$ factor and $G \times G$ acts freely on $R$ with $G$ embedded into $G \times G$ diagonally as above. (This follows from a general theorem on the asymptotic inclusion and the central sequence subfactor claimed in [28, III.2]. See [18] and [21, Appendix] for a complete proof.) It now turns out that the system of the $M_{\infty}-M_{\infty}$ bimodules of the asymptotic inclusion 
$M \vee\left(M^{\prime} \cap M_{\infty}\right) \subset M_{\infty}$ of $N \subset M=N \times G$ is given by the dual of the quantum double of the function algebra $F(G)$ on the group $G$ in the sense of Drinfel'd [8]. (See [4], [5], [6], [7] for results on this system. Also see [23], [24] for operator algebraic study of this type of subfactors arising from finite group actions.) In this sense, if the paragroup is really a (finite) group, the asymptotic inclusion really gives the quantum double. (In the setting of TQFT, this is the Dijkgraaf-Witten construction [7] with trivial 3-cocycle.) So it can be said that the asymptotic inclusion gives the quantum double of a paragroup.

A general paragroup is more like a general Hopf algebra than a quantum group, so that in general the fusion algebra is not commutative and there is no analogue of the $R$-matrix. These inconveniences can be eliminated by passing to the "quantum double",

At the end, we discuss three constructions mentioned in the Introduction. In Section 3, a relation was shown between the TQFT based on triangulation and the asymptotic inclusion. Here in this section a relation between the asymptotic inclusion and the quantum double was observed. Finally we mention an interpretation of Ocneanu's recent announcement on chirality [35] from a viewpoint of a relation between the TQFT based on triangulation and the quantum double.

Conceptually, the quantum "double" construction pairs the original object with something "dual" to the original object, and then appeals to some machinery to produce a higher symmetry. In the ordinary quantum double construction, the "dual" object is the dual Hopf algebra and the "machinery" changes the product structure. In the asymptotic inclusion, the "dual" object is an opposite algebra $M^{\text {opp }} \cong M^{\prime} \cap M_{\infty}$, and the "machinery" allows us to pass from the system of the $M \vee\left(M^{\prime} \cap M_{\infty}\right)$ $M \vee\left(M^{\prime} \cap M_{\infty}\right)$ bimodules to that of the $M_{\infty}-M_{\infty}$ bimodules. In the case of TQFT based on triangulation, Ocneanu introduced a notion of non-degenerate braided system of bimodules in [35], and in that case a factorization of the TQFT holds as a tensor product of the TQFT given by the same system with the Reshetikhin-Turaev type method based on surgery [40] and its complex conjugate. (The ReshetikhinTuraev TQFT [40] gave a rigorous realization of the TQFT predicted by Witten [46].) Ocneanu's non-degenerate bimodule assumption means that the system of bimodules has a high enough symmetry from the beginning, and then the "quantum double" construction is just making everything "double" without any more change. In this sense, the TQFT based triangulation makes everything double if we have a high symmetry at the beginning. In general, we can interpret the TQFT based on triangulation as a method making a "double" and changing the symmetry to a higher one. In this construction, the "dual" object is complex conjugate. This gives a more conceptual understanding of Ocneanu's result [35], which generalized an earlier result of Turaev [41].

We finally mention a relation to RCFT. Ordinary paragroups do not give analogues of RCFT, as shown in [3], but the above shows that after passing to the asymptotic inclusion, the "quantum double for paragroups", we have a symmetry high enough to get an RCFT, as explained in the previous section. 


\section{References}

[1] M. Atiyah, Topological quantum field theories, Publ. Math. IHES 68 (1989), $175-186$.

[2] J. Bion-Nadal, Subfactor of the hyperfinite $I_{1}$ factor with Coxeter graph $E_{6}$ as invariant, J. Operator Theory 28 (1992), 27-50.

[3] J. de Boer \& J. Goeree, Markov traces and $I I_{1}$ factors in conformal field theory, Comm. Math. Phys. 139 (1991), 267-304.

[4] R. Dijkgraaf, C. Vafa, E. Verlinde, \& H. Verlinde, The operator algebra of orbifold models, Comm. Math. Phys. bf 123 (1989), 485-526.

[5] R. Dijkgraaf, V. Pasquier, \& P. Roche, Quasi Hopf algebras, group cohomology and orbifold models, Nucl. Phys. B (Proc. Suppl.) 18B (1990), 60-72.

[6] R. Dijkgraaf, V. Pasquier, \& P. Roche, Quasi-quantum groups related to orbifold models, Proceedings of the International Colloquium on Modern Quantum Field Theory, World Scientific, Singapore, (1991), 375-383.

[7] R. Dijkgraaf \& E. Witten, Topological gauge theories and group cohomology, Comm. Math. Phys. 129 (1990), 393-429.

[8] V. G. Drinfel'd, Quantum groups, Proc. ICM-86, Berkeley, 798-820.

[9] B. Durhuus, H. P. Jakobsen, \& R. Nest, Topological quantum field theories from generalized 6j-symbols, Rev. Math. Phys. 5 (1993), 1-67.

[10] D. E. Evans, Quasi-product states on $C^{*}$-algebras, in "Operator algebras and their connections with topology and ergodic theory", Springer Lecture Notes in Math., 1132 (1985), 129-151.

[11] D. E. Evans \& Y. Kawahigashi, From subfactors to 3-dimensional topological quantum field theories and back - a detailed account of Ocneanu's theory -, to appear in Astérisque.

[12] D. E. Evans \& Y. Kawahigashi, Subfactors and conformal field theory, in "Quantum and non-commutative analysis", 341-369, Kluwer Academic (1993).

[13] M. Izumi, On flatness of the Coxeter graph $E_{8}$, to appear in Pac. J. Math.

[14] V. F. R. Jones, Index for subfactors, Invent. Math. 72 (1983), 1-15.

[15] V. F. R. Jones, A polynomial invariant for knots via von Neumann algebras, Bull. Amer. Math. Soc. 12 (1985), 103-112.

[16] Y. Kawahigashi, On flatness of Ocneanu's connections on the Dynkin diagrams and classification of subfactors, to appear in J. Funct. Anal. 
[17] Y. Kawahigashi, Exactly solvable orbifold models and subfactors, in "Functional Analysis and Related Topics", Lect. Notes in Math. (Springer Verlag) 1540 (1992), 127-147.

[18] Y. Kawahigashi, Centrally trivial automorphisms and an analogue of Connes's $\chi(M)$ for subfactors, Duke Math. J. 71 93-118, (1993).

[19] Y. Kawahigashi, Classification of paragroup actions on subfactors, preprint 1993.

[20] Y. Kawahigashi, Paragroups as quantized Galois groups of subfactors, to appear in Sugaku Exp.

[21] Y. Kawahigashi, Orbifold subfactors, central sequences and the relative Jones invariant $\kappa$, in preparation.

[22] A. N. Kirillov \& N. Yu. Reshetikhin, Representations of the algebra $U_{q}\left(s_{2}\right)$, q-orthogonal polynomials and invariants for links, in "Infinite dimensional Lie algebras and groups" (V. G. Kac, ed.), Adv. Ser. in Math. Phys., vol. 7, 1988, pp. 285-339.

[23] H. Kosaki, A. Munemasa, \& S. Yamagami, On fusion algebras associated to finite group actions, in preparation.

[24] H. Kosaki \& S. Yamagami, Irreducible bimodules associated with crossed product algebras, Internat. J. Math. 3 (1992), 661-676.

[25] G. Moore \& N. Seiberg, Classical and quantum conformal field theory, Comm. Math. Phys. 123 (1989), 177-254.

[26] A. Ocneanu, Quantized group string algebras and Galois theory for algebras, in "Operator algebras and applications, Vol. 2 (Warwick, 1987)," London Math. Soc. Lect. Note Series Vol. 136, Cambridge University Press, 1988, pp. 119-172.

[27] A. Ocneanu, Graph geometry, quantized groups and nonamenable subfactors, Lake Tahoe Lectures, June--July, 1989.

[28] A. Ocneanu, "Quantum symmetry, differential geometry of finite graphs and classification of subfactors", University of Tokyo Seminary Notes 45, (Notes recorded by Y. Kawahigashi), 1991.

[29] A. Ocneanu, An invariant coupling between 3-manifolds and subfactors, with connections to topological and conformal quantum field theory, preprint 1991.

[30] A. Ocneanu, Operator algebras, 3-manifolds and quantum field theory, OHP sheets for the Istanbul talk, July, 1991.

[31] A. Ocneanu, Lectures at Collège de France, Fall 1991. 
[32] A. Ocneanu, Subfactors, topological invariants and roots of unity, talk at the Copenhagen Conference, August, 1992.

[33] A. Ocneanu, Low dimensional operator cohomology, preliminary course notes, 1993.

[34] A. Ocneanu, Seminar talk at University of California, Berkeley, June 1993.

[35] A. Ocneanu, Chirality for operator algebras, (recorded by Y.Kawahigashi), to appear in "Subfactors — Proceedings of the Taniguchi Symposium, Katata, $1993 "$.

[36] S. Popa, Correspondences, preprint, 1986.

[37] S. Popa, Classification of subfactors: reduction to commuting squares, Invent. Math. 101 (1990), 19-43.

[38] S. Popa, Sur la classification des sousfacteurs d'indice fini du facteur hyperfini, C. R. Acad. Sc. Paris 311 (1990), 95-100.

[39] S. Popa, Classification of amenable subfactors of type II, Acta Math. 172 (1994), $253-345$.

[40] N. Yu. Reshetikhin \& V. G. Turaev, Invariants of 3-manifolds via link polynomials and quantum groups, Invent. Math. 103 (1991), 547-597.

[41] V. G. Turaev, Topology of shadows, preprint (1991).

[42] V. G. Turaev \& O. Y. Viro, State sum invariants of 3-manifolds and quantum 6j-symbols, Topology, 31 (1992), 865-902.

[43] C. Vafa, Toward classification of conformal field theories, Phys. Lett. B 206 (1988), 421-426.

[44] E. Verlinde, Fusion rules and modular transformation in $2 D$ conformal field theory, Nucl. Phys. B300 (1988), 360-376.

[45] H. Wenzl, Hecke algebras of type $A$ and subfactors, Invent. Math. 92 (1988), 345-383.

[46] E. Witten, Quantum field theory and Jones polynomial, Comm. Math. Phys. 121 (1989), 351-399.

[47] S. Yamagami, A note on Ocneanu's approach to Jones' index theory, Internat. J. Math. 4 (1993), 859-871. 